Kyrylenko Iryna G., Fediaieva Svitlana I., Miesoiedova Vita, Popadynets' Oleksandr O., Żukow Xawery. Vegetative, metabolic and immune accompaniments of changes in the electrokinetic index of the buccal epithelium under the influence of therapeutic factors. Journal of Education, Health and Sport. 2022;12(1):344-354. eISSN 2391-8306. DOI http://dx.doi.org/10.12775/JEHS.2022.12.01.030 https://apcz.umk.pl/JEHS/article/view/JEHS.2022.12.01.030

https://zenodo.org/record/5992134

The journal has had 40 points in Ministry of Education and Science of Poland parametric evaluation. Annex to the announcement of the Minister of Education and Science of December 21, 2021. No. 32343. Has a Journal's Unique Identifier: 201159. Scientific disciplines assigned: Physical Culture Sciences (Field of Medical sciences and health sciences); Health Sciences (Field of Medical Sciences and Health Sciences).

Punkty Ministerialne z 2019 - aktualny rok 40 punktów. Zalącznik do komunikatu Ministra Edukacji i Nauki z dnia 21 grudnia 2021 r. Lp. 32343. Posiada Unikatowy Identyfikator Czasopisma: 201159. Przypisane dyscypliny naukowe:Nauki o kulturze fizycznej (Dziedzina nauk medyczyych i nauk o zdrowiu); Nauki o zdrowiu (Dziedzina nauk medycznych i nauk o zdrowiu).

(1) The Authors 2022;

This article is published with open access at Licensee Open Journal Systems of Nicolaus Copernicus University in Torun, Polan

Open Access. This article is distributed under the terms of the Creative Commons Attribution Noncommercial License which permits any noncommercial use, distribution, and reproduction in any medium,

provided the original author (s) and source are credited. This is an open access article licensed under the terms of the Creative Commons Attribution Non commercial license Share alike. The authors declare that there is no conflict of interests regarding the publication of this paper.

Received: 02.01.2022. Revised: 17.01.2022. Accepted: 31.01.2022.

\title{
Vegetative, metabolic and immune accompaniments of changes in the electrokinetic index of the buccal epithelium under the influence of therapeutic factors
}

\author{
Iryna G. Kyrylenko ${ }^{1,2}$, Svitlana I. Fediaieva ${ }^{1}$, Vita Miesoiedova ${ }^{3}$, \\ Oleksandr O. Popadynets', Xawery Żukow ${ }^{4}$ \\ ${ }^{1}$ Ukrainian Scientific Research Institute for Medicine of Transport, Odesa, Ukraine \\ iryna2023@gmail.com daddysbestmail@gmail.com \\ ${ }^{2}$ Danylo Halyts'ky̌̌ National Medical University, L'viv, Ukraine \\ svfedyaeva@ukr.net \\ ${ }^{3}$ National Medical University, Ivano-Frankivs'k, Ukraine mva011@gmal.com \\ ${ }^{4}$ Medical University of Bialystok, Bialystok, Poland xaweryzukow@gmail.com
}

\begin{abstract}
s
Background. In previous studies, we have shown that electrokinetic index of buccal epithelium (EKI) correlated with some functional and metabolic parameters. Subsequent studies have shown that changes in EKI correlated with changes in some parameters of EEG, HRV, hemodynamics, metabolism, immunity and fecal microbiocenosis. Further research in this direction was continued on a significantly increased contingent of patients and with the involvement of new methods and factors of influence. This message starts the presentation of the obtained results. Material and methods. Under a observations were 44 men $(49 \pm 15$ years) and 30 women ( $51 \pm 13$ years) without clinical diagnosis or with chronic pyelonephritis in the phase of remission $(23 \mathrm{men})$. We registered caused by the various therapeutic factors changes in EKI, state of the vegetative and hormonal regulation as well as immunity and metabolism, then calculated relationships between changes. Results. In 49 patients the changes in EKI were in the range of $\pm 2,5 \%$, in 19 people EKI increased by more than $2,5 \%$ $(\mathrm{M} \pm \mathrm{SD}=+4,0 \pm 1,6 \%)$, while in 9 people decreased by more than $2,5 \%(-4,2 \pm 1,7 \%)$. The canonical correlation between changes in EKI, on the one hand, and HRV and immunity parameters, on the other, is moderate: $\mathrm{R}=0,478 ; \mathrm{p}=0,023$. The method of discriminant analysis revealed 10 immune and $6 \mathrm{HRV}$ parameters as well as triglycerides and cholesterol, whose changes are characteristic of multidirectional changes in EKI. Conclusion. Electrokinetic index of buccal epithelium responds to therapeutic factors in different directions, accompanied by characteristic changes in a number of parameters of $\mathrm{HRV}$, immunity and metabolism.
\end{abstract}

Keywords: Electrokinetic index, HRV, hormones, immunity, metabolism, relationships. 


\section{INTRODUCTION}

In previous pilot studies, we have shown that electrokinetic index of buccal epithelium (EKI) correlated with some functional and metabolic parameters $[9,18,20]$. Subsequent studies have shown that caused by balneofactors changes in EKI correlated with changes in some parameters of EEG, HRV, hemodynamics and metabolism [1,17] as well as immunity and fecal microbiocenosis [1,19]. Further research in this direction was continued on a significantly increased contingent of patients and with the involvement of new methods and factors of influence. This message starts the presentation of the obtained results.

\section{MATERIALS AND METHODS}

Under a observations were 44 men $(49 \pm 15$ years $)$ and 30 women $(51 \pm 13$ years $)$ without clinical diagnosis or with chronic pyelonephritis in the phase of remission (23 men).

At a receipt, we first determined them the EKI as rate of electronegative nuclei of buccal epithelium by intracellular microelectrophoresis on the device "Biotest" (Kharkiv State University), according to the method described [13,14,22,23,28,30].

Then estimated the state of the autonomous (vegetative) regulation by the method heart rate variability (HRV) [2,5,12,27], using a hardware-programmatic complex "CardioLab+HRV" (KhAI Medica, Kharkiv, Ukraine). Calculated as well Kerdö's Vegetative Index [8].

At last we took venous blood samples for biochemical and immune tests. We determined content in plasma hormones: Cortisol, Testosterone and Triiodothyronine (by the ELISA with the use of analyzer "RT-2100C" and corresponding sets of reagents from "Алкор Био", XEMA Co., Ltd and DRG International Inc.); nitric metabolites: creatinine (by Jaffe's color reaction by Popper's method), urea (urease method by reaction with phenolhypochlorite) and uric acid (uricase method); lipide spectrum of plasma: total cholesterol (by a direct method after the classic reaction by Zlatkis-Zack) and content of him in composition of $\alpha$-lipoproteins (by the enzyme method after precipitation of not $\alpha$-lipoproteins); prae- $\beta$-lipoproteins (expected by the level of triglycerides, by a certain meta-periodate method); $\beta$-lipoproteins (expected by a difference between a total cholesterol and cholesterol in composition $\alpha$-and prae- $\beta$ lipoproteins). The analysis carried out according to instructions [10] with the use of analyzers "Reflotron" (BRD) and "Pointe-180" (USA) and corresponding sets of reagents.

Immune status evaluated on a set of I and II levels recommended by the WHO as described in the manual [21]. For phenotyping subpopulations of lymphocytes used the methods of rosette formation with sheep erythrocytes on which adsorbed monoclonal antibodies against receptors CD3, CD4, CD8, CD22 and CD56 from company "Granum" (Kharkiv) with visualization under light microscope with immersion system. Subpopulation of $\mathrm{T}$ cells with receptors high affinity determined by test of "active" rosette formation. The state of humoral immunity judged by the concentration in serum of Immunoglobulins classes G, A, M (ELISA, analyser "Immunochem", USA) and circulating immune complexes (by polyethylene glycol precipitation method) as well as C-reactive protein (by the ELISA with the use of analyzer "RT-2100C"), Interleukin $1 \beta$ and 6 (ELISA, analyzer "Stat Fax 303", USA, reagents from "Vector-Best", RF).

In portion of capillary the blood we counted up Leukocytogram and calculated its Adaptation Index-2 as well as Strain Index-2 by IL Popovych [4,11,24,25]:

PSI-2 $=\left[(\text { Eosinoph } / 2,75-1)^{2}+(\text { StubNeutroph } / 4,25-1)^{2}+(\text { Monoc } / 6-1)^{2}+(\text { Leukocyt } / 5-1)^{2}\right] / 4$

We calculated also the Entropy (h) of Immunocytogram (ICG) and Leukocytogram (LCG) using IL Popovych's formula [11] based on classical CE Shannon's formula [29]:

$\mathrm{hICG}=-\left[\mathrm{CD} 4 \cdot \log _{2} \mathrm{CD} 4+\mathrm{CD} 8 \bullet \log _{2} \mathrm{CD} 8+\mathrm{CD} 22 \cdot \log _{2} \mathrm{CD} 22+\mathrm{CD} 16 \bullet \log _{2} \mathrm{CD} 16\right] / \log _{2} 4$

$\mathrm{hLCG}=-\left[\mathrm{L} \bullet \log _{2} \mathrm{~L}+\mathrm{M} \bullet \log _{2} \mathrm{M}+\mathrm{E} \bullet \log _{2} \mathrm{E}+\mathrm{SNN} \bullet \log _{2} \mathrm{SNN}+\mathrm{StubN} \bullet \log _{2} \mathrm{StubN}\right] / \log _{2} 5$ 
Parameters of phagocytic function of neutrophils estimated as described by SD Douglas and PG Quie [7] with moderately modification by MM Kovbasnyuk [16]. The objects of phagocytosis served daily cultures of Staphylococcus aureus (ATCC N 25423 F49) as typical specimen for Gram-positive Bacteria and Escherichia coli (O55 K59) as typical representative of Gram-negative Bacteria. Both cultures obtained from Laboratory of Hydro-Geological Regime-Operational Station JSC "Truskavets'kurort". Take into account the following parameters of Phagocytosis: activity (percentage of neutrophils, in which found microbes Hamburger's Phagocytic Index PhI), intensity (number of microbes absorbed one phagocytes - Microbial Count MC or Right's Index) and completeness (percentage of dead microbes Killing Index KI). On the basis of the recorded partial parameters of Phagocytosis, taking into account the Neutrophils (N) content of 1 L blood, we calculated the integral parameter Bactericidal Capacity of Neutrophils (BCCN) by the formula [11]:

$\operatorname{BCCN}\left(10^{9} \mathrm{Bact} / \mathrm{L}\right)=\mathrm{N}\left(10^{9} / \mathrm{L}\right) \cdot \mathrm{PhI}(\%) \cdot \mathrm{MC}(\mathrm{Bact} / \mathrm{Phag}) \cdot \mathrm{KI}(\%) \bullet 10^{-4}$

After therapy: drinking of bioactive water Naftussya only $(n=22)$, Naftussya and applications of ozokerite and mineral pools $(n=13)$ or electrostimulation by device "VEB"[1] $(\mathrm{n}=39)$, all testes repeated.

For statistical analysis used the software package "Statistica 64".

\section{RESULTS AND DISCUSSION}

In this sample, as in previous ones, a variety of changes in EKI were found. In 49 patients the changes were in the range of $\pm 2,5 \%$ and were considered insignificant, in 19 people EKI increased by more than $2,5 \%(\mathrm{M} \pm \mathrm{SD}=+4,0 \pm 1,6 \%)$, while in 9 people decreased by more than $2,5 \%(-4,2 \pm 1,7 \%)$. All three variants were observed in response to all three treatment regimens. This is consistent with the well-documented concept of the multivariate effect of therapeutic factors of the spa of Truskavets' on the parameters of the human and animal body due to individual reactivity [3,6,15,16,26,31-33].

According to the formula:

$|r| \geq\left\{\exp \left[2 t /(n-1,5)^{0,5}\right]-1\right\} /\left\{\exp \left[2 t /(n-1,5)^{0,5}\right]+1\right\}$,

for a sample of 74 observations critical value of correlation coefficient module $|r|$ at $\mathrm{p}<0,05$ $(\mathrm{t}>2,00)$ is 0,23 ; at $\mathrm{p}<0,01(\mathrm{t}>2,66)$ is 0,30 ; at $\mathrm{p}<0,001(\mathrm{t}>3,46)$ is 0,38 .

Screening of correlations between changes in EKI and recorded parameters showed a direct correlation with phagocytosis activity by neutrophils vs E. coli, while inverse correlation with eosinophilia, leukocytogram strain index, and a number of HRV parameters (Table 1).

Table 1. Factor loads and correlations for change in HRV\&Immunity parameters and Electrokinetic Index

\begin{tabular}{|c|c|c|}
\hline Left set & $\mathbf{R}$ & $\mathbf{r}$ \\
\hline Eosinophiles, \% & 0,747 & $-0,36$ \\
\hline Total Power HRV, msec ${ }^{2}$ & 0,542 & $-0,26$ \\
\hline (VLF+ULF) HRV PS, msec ${ }^{2}$ & 0,532 & $-0,25$ \\
\hline Popovych's Strain Index-2 & 0,506 & $-0,24$ \\
\hline ULF HRV PS, msec $^{2}$ & 0,504 & $-0,24$ \\
\hline $\mathrm{LF} /(\mathrm{LF}+\mathrm{HF})$ & 0,483 & $-0,23$ \\
\hline $\mathrm{LF} / \mathrm{HF}$ & 0,475 & $-0,23$ \\
\hline Phagocytose Index vs E. coli, \% & $-0,535$ & $\mathbf{0 , 2 6}$ \\
\hline Right set & $\mathbf{R}$ & \\
\hline Electrokinetic Index, \% & -1 & \\
\hline
\end{tabular}

If the $\mathrm{LF} /(\mathrm{LF}+\mathrm{HF})$ and $\mathrm{LF} / \mathrm{HF}$ indices uniquely reflect sympathetic tone and sympathetic-vagal balance, respectively, VLF and ULF bands still do not have a clear 
physiological interpretation. Let's take advantage of the latest wonderful review Shaffer F \& Ginsberg JP [27]. We quote. "There is uncertainty regarding the physiological mechanisms responsible for activity within the VLF $(0,04 \div 0,0033 \mathrm{~Hz})$ band. The heart's intrinsic nervous system appears to contribute to the VLF rhythm and the sympathetic nervous system influences the amplitude and frequency of its oscillations. Very-low-frequency power may also be generated by physical activity, thermoregulatory, renin-angiotensin, and endothelial influences on the heart. Vagal activity may contribute to VLF power since parasympathetic blockade almost completely abolishes it. In contrast, sympathetic blockade does not affect VLF power and VLF activity is seen in tetraplegics, whose sympathetic nervous system innervation of the heart and lungs is disrupted. The VLF rhythm appears to be generated by the stimulation of afferent sensory neurons in the heart. This, in turn, activates various levels of the feedback and feed-forward loops in the heart's intrinsic cardiac nervous system, as well as between the heart, the extrinsic cardiac ganglia, and spinal column. This experimental evidence suggests that the heart intrinsically generates the VLF rhythm and efferent sympathetic nervous system activity due to physical activity and stress responses modulates its amplitude and frequency".

Because in our device ULF band (range $0,015 \div 0,003 \mathrm{~Hz}$ ) is integrated into the lower zone of VLF band, what has been said about the latter also applies to the former. By the way, the relative power spectrum of these bands during the analysis were combined into an option (VLF+ULF). The presence in the factor structure of total power of HRV is explained by the fact that the shares of VLF and ULF bands are on average $47 \%$ and $6 \%$, respectively.

The canonical correlation between changes in EKI, on the one hand, and HRV and immunity parameters, on the other, is moderate (Fig. 1).

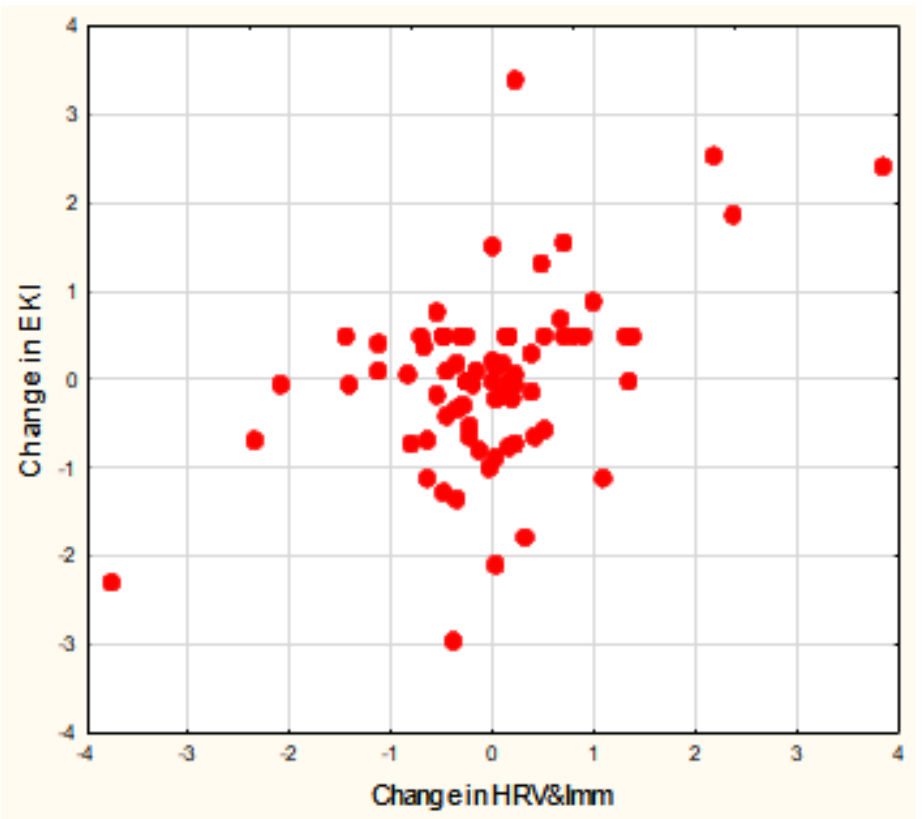

$\mathrm{R}=0,478 ; \mathrm{R}^{2}=0,229 ; \chi_{(8)}^{2}=17,7 ; \mathrm{p}=0,023 ; \Lambda$ Prime $=0,771$

Fig. 1. Scatterplot of canonical correlation between change in HRV and Immune parameters (X-line) and Electrokinetic index (Y-line)

Another approach to identifying autonomic, hormonal, immune, and metabolic accompaniments to EKI changes is discriminant analysis. The forward stepwise program included 19 parameters in the discriminant model. These are, in addition to EKI by definition, 10 immune and $6 \mathrm{HRV}$ parameters as well as triglycerides and cholesterol levels standardized by sex and age (Tables 2 and 3 ). 
Table 2. Discriminant Function Analysis Summary for Changes in HRV, Immune and Metabolic Variables

Step 19, $N$ of vars in model: 19; Grouping: 3 grps. Wilks' $\Lambda$ : 0,0546; approx. $F_{(38)}=9,1 ; p<10^{-6}$

\begin{tabular}{|c|c|c|c|c|c|c|c|c|}
\hline $\begin{array}{l}\text { Variables } \\
\text { change in which } \\
\text { currently in the model }\end{array}$ & $\begin{array}{l}\text { EKI } \\
>+2,5 \% \\
(19)\end{array}$ & $\begin{array}{l}\text { EKI } \\
\pm 2,5 \\
(49)\end{array}$ & $\begin{array}{l}\text { EKI } \\
<-2,5 \% \\
(6)\end{array}$ & $\begin{array}{l}\text { Wilks } \\
\Lambda\end{array}$ & $\begin{array}{l}\text { Parti- } \\
\text { al } \Lambda\end{array}$ & $\begin{array}{l}\text { F-re- } \\
\text { move } \\
(2,53)\end{array}$ & $\begin{array}{l}\text { p- } \\
\text { level }\end{array}$ & $\begin{array}{l}\text { Tole- } \\
\text { rancy }\end{array}$ \\
\hline Electrokinetic Index, \% & $+4,0$ & $+0,65$ & $-4,2$ & 0,299 & 0,183 & 118 & $10^{-6}$ & 0,509 \\
\hline Popovych's Adaptation Index-2 & $+0,28$ & $+0,11$ & $-0,16$ & 0,060 & 0,904 & 2,83 & 0,068 & 0,657 \\
\hline Polymorphonucl Neutrophils, \% & $+1,2$ & $-0,7$ & $-2,7$ & 0,066 & 0,824 & 5,67 & 0,006 & 0,175 \\
\hline Bactericidity vs E. coli, $10^{9} \mathrm{~B} / \mathrm{L}$ & +18 & +13 & +5 & 0,060 & 0,906 & 2,75 & 0,073 & 0,439 \\
\hline Phagocytose Index vs E. coli, \% & $+0,1$ & 0,0 & $-1,9$ & 0,063 & 0,867 & 4,05 & 0,023 & 0,597 \\
\hline Interleukin-6, ng/L & $-0,15$ & $-0,02$ & $-0,78$ & 0,060 & 0,914 & 2,50 & 0,092 & 0,424 \\
\hline Eosinophils, \% & $-0,34$ & $-0,24$ & $+2,7$ & 0,060 & 0,911 & 2,60 & 0,084 & 0,524 \\
\hline Leukocytes, $10^{9} / \mathrm{L}$ & $-0,04$ & $-0,19$ & $+0,65$ & 0,057 & 0,964 & 0,99 & 0,379 & 0,555 \\
\hline Immunocytogram Entropy & -11 & +4 & +4 & 0,080 & 0,686 & 12,1 & $10^{-4}$ & 0,363 \\
\hline$\left(\right.$ VLF+ULF) HRV PS, msec $^{2}$ & -2604 & -335 & -419 & 0,056 & 0,967 & 0,89 & 0,416 & 0,750 \\
\hline Mode HRV, msec & -53 & -46 & -20 & 0,072 & 0,763 & 8,25 & 0,001 & 0,467 \\
\hline Baevsky's Stress Index, units & +31 & -11 & +226 & 0,071 & 0,773 & 7,76 & 0,001 & 0,044 \\
\hline AMo/MxDMn Index, units & +50 & -17 & +207 & 0,068 & 0,803 & 6,49 & 0,003 & 0,053 \\
\hline Baevsky's ARS Index, units & $+0,16$ & $-0,13$ & $+1,53$ & 0,058 & 0,947 & 1,49 & 0,235 & 0,474 \\
\hline ULF HRV PS, \% & $+0,1$ & $-0,7$ & $+6,4$ & 0,056 & 0,979 & 0,58 & 0,565 & 0,636 \\
\hline Triglycerides standardized, Z & $-0,33$ & $+0,14$ & $-0,62$ & 0,058 & 0,943 & 1,60 & 0,211 & 0,779 \\
\hline Cholesterol standardized, Z & $-0,37$ & $-0,21$ & $-0,45$ & 0,065 & 0,837 & 5,17 & 0,009 & 0,375 \\
\hline 0 Lymphocytes, \% & $-3,4$ & $-0,7$ & $-2,7$ & 0,080 & 0,684 & 12,2 & $10^{-4}$ & 0,386 \\
\hline Pan Lymphocytes, \% & $-1,3$ & $+0,6$ & $-0,3$ & 0,059 & 0,927 & 2,09 & 0,133 & 0,193 \\
\hline
\end{tabular}

Table 3. Summary of Stepwise Analysis for Changes in HRV, Immune and Metabolic Variables, ranked by criterion Lambda

\begin{tabular}{|l|l|l|l|l|l|}
\hline $\begin{array}{l}\text { Variables currently } \\
\text { in the model }\end{array}$ & $\begin{array}{l}\text { F to } \\
\text { enter }\end{array}$ & $\begin{array}{l}\text { p- } \\
\text { level }\end{array}$ & $\Lambda$ & $\begin{array}{l}\text { F-va- } \\
\text { lue }\end{array}$ & $\begin{array}{l}\text { p- } \\
\text { level }\end{array}$ \\
\hline Electrokinetic Index, \% & 121 & $10^{-6}$ & 0,227 & 121 & $10^{-6}$ \\
\hline Phagocytose Index vs E. coli, \% & 5,60 & 0,006 & 0,195 & 44,2 & $10^{-6}$ \\
\hline Baevsky's Stress Index, units & 4,81 & 0,011 & 0,171 & 32,6 & $10^{-6}$ \\
\hline Leukocytes, 10 $/ \mathbf{L}$ & 3,13 & 0,050 & 0,157 & 25,9 & $10^{-6}$ \\
\hline ULF HRV PS, \% & 2,65 & 0,078 & 0,145 & 21,7 & $10^{-6}$ \\
\hline 0 Lymphocytes, \% & 2,34 & 0,105 & 0,136 & 18,8 & $10^{-6}$ \\
\hline Immunocytogram Entropy & 5,31 & 0,007 & 0,117 & 17,9 & $10^{-6}$ \\
\hline Mode HRV, msec & 3,36 & 0,041 & 0,106 & 16,6 & $10^{-6}$ \\
\hline AMo/MxDMn Index, units & 2,42 & 0,097 & 0,098 & 15,3 & $10^{-6}$ \\
\hline (VLF+ULF) HRV PS, msec ${ }^{2}$ & 1,60 & 0,210 & 0,093 & 14,1 & $10^{-6}$ \\
\hline Bactericidity vs E. coli, 10 Bac/L & 1,29 & 0,284 & 0,090 & 13,0 & $10^{-6}$ \\
\hline Polymorphonucl Neutrophils, \% & 2,15 & 0,125 & 0,084 & 12,3 & $10^{-6}$ \\
\hline Baevsky's ARS Index, units & 1,77 & 0,179 & 0,079 & 11,6 & $10^{-6}$ \\
\hline Cholesterol standardized, Z & 1,94 & 0,152 & 0,074 & 11,1 & $10^{-6}$ \\
\hline Interleukin-6, ng/L & 1,56 & 0,218 & 0,070 & 10,6 & $10^{-6}$ \\
\hline Eosinophiles, \% & 1,50 & 0,232 & 0,066 & 10,1 & $10^{-6}$ \\
\hline Popovych's Adaptation Index-2 & 1,40 & 0,255 & 0,063 & 9,63 & $10^{-6}$ \\
\hline Pan Lymphocytes, \% & 2,47 & 0,094 & 0,058 & 9,46 & $10^{-6}$ \\
\hline Triglycerides standardized, Z & 1,60 & 0,211 & 0,055 & 9,15 & $10^{-6}$ \\
\hline
\end{tabular}

The rest of the registered parameters were outside the model, despite the fact that some of them carry identifying information (Table 4). 
Table 4. Discriminant Function Analysis Summary. HRV, Endocrine, Immune and Metabolic Variables change in which currently not in the model

\begin{tabular}{|c|c|c|c|c|c|c|c|c|}
\hline Variables & $\begin{array}{l}\text { EKI } \\
>+2,5 \\
\%\end{array}$ & $\begin{array}{l}\text { EKI } \\
\pm 2,5 \\
\%\end{array}$ & $\begin{array}{l}\text { EKI } \\
<-2,5 \\
\%\end{array}$ & $\begin{array}{l}\text { Wilks } \\
\Lambda\end{array}$ & $\begin{array}{l}\text { Parti- } \\
\text { al } \Lambda\end{array}$ & $\begin{array}{l}\mathrm{F} \text { to } \\
\text { enter }\end{array}$ & $\begin{array}{l}\mathrm{p}- \\
\text { level }\end{array}$ & $\begin{array}{l}\text { Tole- } \\
\text { rancy }\end{array}$ \\
\hline Uric acid standardized, $\mathrm{Z}$ & $+0,08$ & $+0,04$ & $-0,01$ & 0,054 & 0,993 & 0,193 & 0,825 & 0,763 \\
\hline Interleukin-1, ng/L & $+0,02$ & $-0,27$ & $-0,65$ & 0,055 & 0,999 & 0,016 & 0,984 & 0,679 \\
\hline $\mathrm{CD3}^{+} \mathrm{T}$ active Lymphocytes, \% & $+1,4$ & $-0,3$ & $-0,5$ & 0,054 & 0,991 & 0,228 & 0,797 & 0,681 \\
\hline Immunoglobulins $\mathrm{M}, \mathrm{g} / \mathrm{L}$ & $-0,03$ & $-0,03$ & $-0,20$ & 0,054 & 0,989 & 0,294 & 0,746 & 0,827 \\
\hline Micr. Count vs St. aur., Bac/Phag & $+5,3$ & $+2,7$ & $+1,2$ & 0,053 & 0,978 & 0,598 & 0,553 & 0,721 \\
\hline Micr. Count vs E. coli, Bact/Phag & $+4,1$ & $+2,5$ & 0,0 & 0,054 & 0,992 & 0,204 & 0,816 & 0,480 \\
\hline 100(1-Pd/HR) as Kerdö Index, \% & $+7,0$ & $+5,8$ & $+2,1$ & 0,054 & 0,982 & 0,519 & 0,594 & 0,536 \\
\hline Stub Neutrophils, \% & $+0,6$ & 0,0 & $-0,2$ & 0,054 & 0,989 & 0,284 & 0,754 & 0,703 \\
\hline Cortisol, nM/L & +28 & +27 & -41 & 0,053 & 0,978 & 0,598 & 0,553 & 0,721 \\
\hline Mx & -8 & -7 & -46 & 0,054 & 0,979 & 0,546 & 0,582 & 0,629 \\
\hline Triiodothyronin & $-0,17$ & $-0,05$ & $-0,16$ & 0,054 & 0,978 & 0,598 & 0,746 & 0,827 \\
\hline VLF HRV PS, $\%$ & $-0,2$ & $-2,5$ & -23 & 0,054 & 0,982 & 0,464 & 0,631 & 0,541 \\
\hline Urea, mM/L & $-0,82$ & $-0,10$ & $+0,15$ & 0,054 & 0,985 & 0,228 & 0,412 & 0,659 \\
\hline Imn & $+0,66$ & $+0,94$ & $+1,36$ & 0,053 & 0,966 & 0,903 & 0,412 & 0,779 \\
\hline Imm & $-0,09$ & $-0,07$ & $-0,05$ & 0,054 & 0,997 & 0,088 & 0,916 & 0,659 \\
\hline Mon & $-0,1$ & $+0,3$ & $+0,5$ & 0,054 & 0,994 & 0,151 & 0,861 & 0,327 \\
\hline Leuk & -2 & +3 & +29 & 0,055 & 0,999 & 0,032 & 0,969 & 0,169 \\
\hline Popov & $-0,03$ & $-0,02$ & $+0,49$ & 0,054 & 0,980 & 0,519 & 0,598 & 0,536 \\
\hline V PS, \% & $-1,4$ & $+1,2$ & $+18,3$ & & 0,990 & 0,256 & 0,775 & 0,650 \\
\hline $\mathrm{LF} /(\mathrm{LF}+\mathrm{HF}), \%$ & -3 & -3 & +12 & 0,054 & 0,990 & 0,525 & 0,594 & 0,655 \\
\hline $\mathrm{LF} / \mathrm{I}$ & $-0,3$ & $-0,7$ & $+5,7$ & 0,054 & 0,990 & 0,903 & 0,412 & 0,779 \\
\hline LF HRV & -608 & -159 & -28 & 0,053 & 0,978 & 0,575 & 0,566 & 0,796 \\
\hline SDNN, ms & -14 & -3 & -9 & 0,054 & 0,995 & 0,128 & 0,880 & 0,117 \\
\hline RMSS & $-6,0$ & $-1,8$ & $-6,3$ & 0,054 & 0,992 & 0,209 & 0,812 & 0,818 \\
\hline HF HRV PS, & -177 & -11 & -115 & 0,054 & 0,980 & 0,525 & 0,594 & 0,753 \\
\hline C-Reactive $\operatorname{Pr}$ & $-0,28$ & $-0,15$ & $-0,35$ & 0,054 & 0,992 & 0,220 & 0,803 & 0,012 \\
\hline CD56 $^{+}$NK Lymph & $-2,0$ & $-0,6$ & $-3,3$ & 0,055 & 0,999 & 0,032 & 0,968 & 0,729 \\
\hline Phagocytose Ind vs & $-0,3$ & $+0,2$ & $-0,5$ & 0,054 & 0,994 & 0,163 & 0,850 & 0,717 \\
\hline CIC, units & $-0,4$ & $+5,5$ & $-1,2$ & 0,054 & 0,990 & 0,265 & 0,768 & 0,717 \\
\hline Killing Ind & $+5,2$ & $+6,6$ & $+5,3$ & 0,054 & 0,983 & 0,450 & 0,640 & 0,388 \\
\hline Killing Index vs E. coli, \% & $+3,7$ & $+6,9$ & $+2,5$ & 0,055 & 0,999 & 0,013 & 0,987 & 0,390 \\
\hline CD4 $^{+}$T-helper Lymphocytes, \% & $+1,1$ & $-0,45$ & $+1,6$ & & 0,985 & 0,386 & 0,682 & 0,726 \\
\hline Testosterone normalized, $\mathrm{Z}$ & $-0,28$ & $-0,46$ & $-0,18$ & 0,054 & 0,992 & 0,209 & 0,812 & 0,818 \\
\hline $\mathrm{CD22}^{+}$B Lymphocytes, \% & $+2,6$ & $+0,7$ & $+1,5$ & 0,054 & 0,992 & 0,205 & 0,815 & 0,112 \\
\hline CD8 $^{+}$T-cytolytic Lymphocyt & $+1,4$ & $+1,1$ & $+2,9$ & 0,055 & 0,999 & 0,025 & 0,975 & 0,764 \\
\hline Bactericidity vs St. aur., $10^{9} \mathrm{Bac} / \mathrm{L}$ & +22 & +12 & +20 & 0,054 & 0,995 & 0,123 & 0,885 & 0,277 \\
\hline AMo HRV, \% & $+5,5$ & $-0,2$ & $+8,9$ & 0,053 & 0,979 & 0,564 & 0,572 & 0,601 \\
\hline
\end{tabular}

Next, the 19-dimensional space of discriminant variables transforms into 2-dimensional space of a canonical roots. The canonical correlation coefficient is for Root 10,950 (Wilks' $\Lambda=0,055 ; \chi_{(38)}^{2}=180 ; \mathrm{p}<10^{-6}$ ), for Root 20,665 (Wilks' $\Lambda=0,558 ; \chi_{(18)}^{2}=36,2 ; \mathrm{p}=0,007$ ). The major root contains $92 \%$ of discriminative capabiliities, while the minor $8 \%$ only. The calculation of the discriminant root values for each person as the sum of the products of raw coefficients to the individual values of discriminant variables together with the constant (Table 5) enables the visualization of each patient in the information space of the roots (Fig. 2). 
Table 5. Standardized and Raw Coefficients and Constants for Neuro-Immune Variables

\begin{tabular}{|c|c|c|c|c|}
\hline Coefficients & \multicolumn{2}{|c|}{ Standardized } & \multicolumn{2}{|c|}{ Raw } \\
\hline Variables currently in the model & Root 1 & Root 2 & Root 1 & Root 2 \\
\hline Electrokinetic Index, \% & $-1,333$ & 0,078 & $-1,136$ & 0,067 \\
\hline Phagocytose Index vs E. coli, \% & $-0,346$ & $-0,507$ & $-0,310$ & $-0,454$ \\
\hline Baevsky's Stress Index, units & 2,380 & 0,411 & 0,014 & 0,002 \\
\hline Leukocytes, $10^{9} / \mathrm{L}$ & $-0,093$ & 0,359 & $-0,080$ & 0,307 \\
\hline ULF HRV PS, $\%$ & 0,151 & 0,171 & 0,019 & 0,022 \\
\hline 0 Lymphocytes, \% & 0,784 & $-0,770$ & 0,130 & $-0,128$ \\
\hline Immunocytogram Entropy & 0,834 & $-0,730$ & 0,025 & $-0,022$ \\
\hline Mode HRV, msec & 0,750 & 0,019 & 0,0059 & 0,0001 \\
\hline AMo/MxDMn Index, units & $-2,034$ & 0,160 & $-0,0099$ & 0,0008 \\
\hline$(\mathrm{VLF}+\mathrm{ULF}) \mathrm{HRV}$ PS, msec ${ }^{2}$ & $-0,146$ & $-0,234$ & $-0,00004$ & $-0,00006$ \\
\hline Bactericidity vs E. coli, $10^{9}$ Bacteria/L & 0,460 & $-0,232$ & 0,0136 & $-0,0069$ \\
\hline Polymorphonucl Neutrophils, \% & $-1,033$ & 0,324 & $-0,176$ & 0,056 \\
\hline Baevsky's ARS Index, units & $-0,349$ & $-0,077$ & $-0,126$ & $-0,028$ \\
\hline Cholesterol standardized, Z & $-0,695$ & $-0,001$ & $-0,929$ & $-0,002$ \\
\hline Interleukin-6, ng/L & 0,471 & $-0,084$ & 0,376 & $-0,067$ \\
\hline Eosinophiles, \% & $-0,396$ & 0,254 & $-0,213$ & 0,137 \\
\hline Popovych's Adaptation Index-2, points & $-0,388$ & 0,156 & $-0,662$ & 0,266 \\
\hline Panlymphocytes, \% & $-0,647$ & $-0,065$ & $-0,130$ & $-0,013$ \\
\hline \multirow[t]{3}{*}{ Triglycerides standardized, $\mathrm{Z}$} & 0,111 & $-0,375$ & 0,126 & $-0,426$ \\
\hline & \multicolumn{2}{|c|}{ Constants } & 1,220 & $-0,332$ \\
\hline & \multicolumn{2}{|c|}{\begin{tabular}{|c|} 
Eigenvalues \\
\end{tabular}} & 9,208 & 0,793 \\
\hline \multicolumn{3}{|c|}{ Cumulative Proportion } & 0,921 & 1 \\
\hline
\end{tabular}

Table 6 presents the full Structural coefficients and average values (centroids) of Roots as well as changes in Variables, both included and not included in the model.

Table 6. Correlations Variables-Canonical Roots, Means of Roots and Changes in Variables

\begin{tabular}{|c|c|c|c|c|c|}
\hline \multirow{2}{*}{$\begin{array}{l}\text { Change in Variables } \\
\text { Root } 1(92 \%)\end{array}$} & \multicolumn{2}{|c|}{$\begin{array}{c}\text { Correlations } \\
\text { Variables-Roots }\end{array}$} & \multirow{2}{*}{$\begin{array}{l}\text { EKI } \\
>+2,5 \% \\
-4,01\end{array}$} & \multirow{2}{*}{$\begin{array}{l}\text { EKI } \\
\pm 2,5 \% \\
+0,65\end{array}$} & \multirow{2}{*}{$\begin{array}{l}\text { EKI } \\
<-2,5 \% \\
+7,38\end{array}$} \\
\hline & Root 1 & Root 2 & & & \\
\hline Electrokinetic Index, \% & $-0,609$ & $-0,011$ & $+4,0$ & $+0,65$ & $-4,2$ \\
\hline Popovych's Adaptation Index-2, points & $-0,065$ & $-0,014$ & $+0,28$ & $+0,11$ & $-0,16$ \\
\hline Polymorphonucleary Neutrophils, \% & $-0,060$ & 0,022 & $+1,2$ & $-0,7$ & $-2,7$ \\
\hline Stub Neutrophils, $\%$ & & & $+0,6$ & 0,0 & $-0,2$ \\
\hline $\mathrm{CD3}^{+} \mathrm{T}$ active Lymphocytes, \% & & & $+1,4$ & $-0,3$ & $-0,5$ \\
\hline Bactericidity vs E. coli, $10^{9}$ Bacteria/L & $-0,032$ & $-0,009$ & +18 & +13 & +5 \\
\hline Phagocytose Index vs E. coli, \% & $-0,121$ & $-0,348$ & $+0,1$ & 0,0 & $-1,9$ \\
\hline Interleukin-6, ng/L & $-0,029$ & $-0,162$ & $-0,15$ & $-0,02$ & $-0,78$ \\
\hline Interleukin-1, ng/L & & & $+0,02$ & $-0,27$ & $-0,65$ \\
\hline Eosinophils, \% & 0,111 & 0,313 & $-0,34$ & $-0,24$ & $+2,7$ \\
\hline Leukocytes, $10^{9} / \mathrm{L}$ & 0,033 & 0,192 & $-0,04$ & $-0,19$ & $+0,65$ \\
\hline Monocytes, \% & & & $-0,1$ & $+0,3$ & $+0,5$ \\
\hline Immunocytogram Entropy & 0,052 & $-0,139$ & -11 & +4 & +4 \\
\hline Leukocytogram Entropy & & & -2 & +3 & +29 \\
\hline Popovych's Strain Index-2, points & & & $-0,03$ & $-0,02$ & $+0,49$ \\
\hline LF HRV PS, \% & & & $-1,4$ & $+1,2$ & $+18,3$ \\
\hline
\end{tabular}




\begin{tabular}{|c|c|c|c|c|c|}
\hline $\mathrm{LF} /(\mathrm{LF}+\mathrm{HF}), \%$ & & & -3 & -3 & +12 \\
\hline Urea, mM/L & & & $-0,82$ & $-0,10$ & $+0,15$ \\
\hline$(\mathrm{VLF}+\mathrm{ULF}) \mathrm{HRV}$ PS, $\mathrm{msec}^{2}$ & 0,043 & $-0,120$ & -2604 & -335 & -419 \\
\hline LF HRV PS, msec ${ }^{2}$ & & & -608 & -159 & -28 \\
\hline Mode HRV, msec & 0,020 & 0,029 & -53 & -46 & -20 \\
\hline Baevsky's Stress Index, units & 0,066 & 0,369 & +31 & -11 & +226 \\
\hline AMo/MxDMn Index, units & 0,036 & 0,329 & +50 & -17 & +207 \\
\hline Baevsky's ARS Index, units & 0,029 & 0,158 & $+0,16$ & $-0,13$ & $+1,53$ \\
\hline ULF HRV PS, \% & 0,049 & 0,220 & $+0,1$ & $-0,7$ & $+6,4$ \\
\hline Root $2(8 \%)$ & Root 1 & Root 2 & $+0,90$ & $-0,59$ & $+1,98$ \\
\hline Triglycerides standardized, $\mathrm{Z}$ & 0,005 & $-0,343$ & $-0,33$ & $+0,14$ & $-0,62$ \\
\hline Cholesterol standardized, $\mathrm{Z}$ & 0,003 & $-0,135$ & $-0,37$ & $-0,21$ & $-0,45$ \\
\hline 0 Lymphocytes, \% & 0,029 & $-0,204$ & $-3,4$ & $-0,7$ & $-2,7$ \\
\hline Pan Lymphocytes, \% & 0,032 & $-0,152$ & $-1,3$ & $+0,6$ & $-0,3$ \\
\hline C-Reactive Protein, mg/L & & & $-0,28$ & $-0,15$ & $-0,35$ \\
\hline CD56 $^{+}$NK Lymphocytes, \% & & & $-2,0$ & $-0,6$ & $-3,3$ \\
\hline SDNN, msec & & & -14 & -3 & -9 \\
\hline RMSSD HRV, msec & & & $-6,0$ & $-1,8$ & $-6,3$ \\
\hline HF HRV PS, msec $^{2}$ & & & -177 & -11 & -115 \\
\hline Amplitude of Mode HRV, \% & & & $+5,5$ & $-0,2$ & $+8,9$ \\
\hline CD4 $^{+}$T-helper Lymphocytes, \% & & & $+1,1$ & $-0,45$ & $+1,6$ \\
\hline CD22 $^{+}$B Lymphocytes, \% & & & $+2,6$ & $+0,7$ & $+1,5$ \\
\hline Bactericidity vs St. aur., $10^{9} \mathrm{Bac} / \mathrm{L}$ & & & +22 & +12 & +20 \\
\hline
\end{tabular}

Fig. 2 shows the data in table. 6, the therapeutic factor-induced increase in EKI is accompanied by an increase in the adaptation index and physiologically favorable or minimal changes in immunity and autonomic regulation, whereas in rare cases a decrease in EKI is also reduced in combination with adverse changes in immunity and autonomic regulation. Individuals with minor changes in EKI occupy an intermediate position along the axis of the first root.

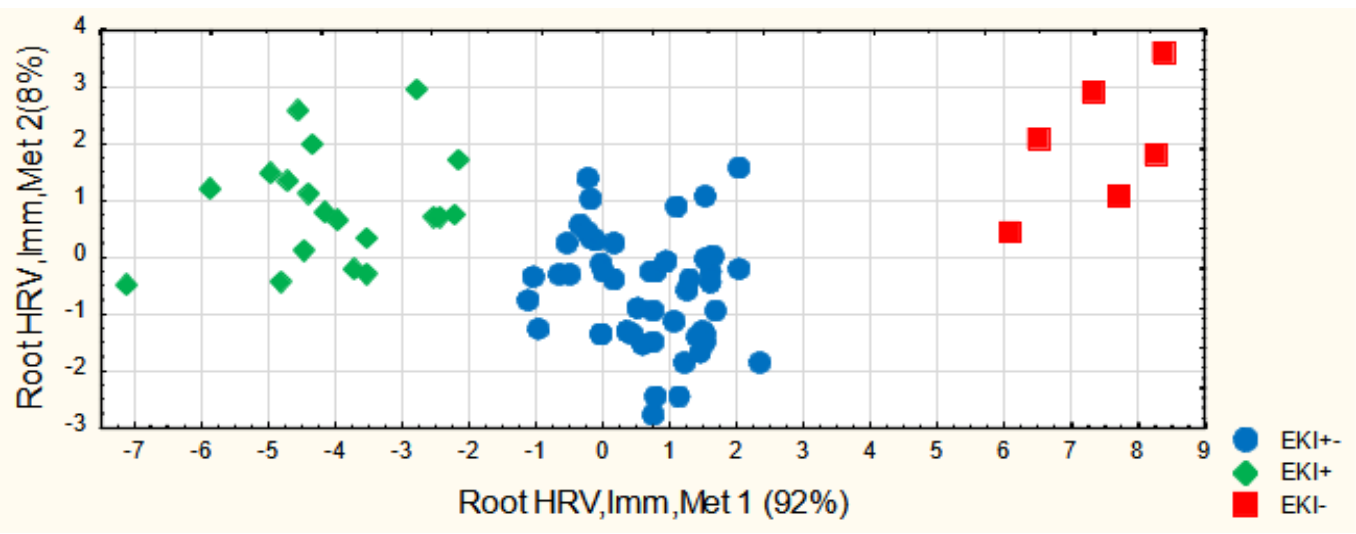

Fig. 2. Individual values of discriminant roots of changes in parameters that are characteristic of different variants of changes in the electrokinetic index

Additional delimitation of clusters occurs along the axis of the second root. The lowest position of persons with insignificant changes in EKI reflects minimal changes in parameters, while a significant increase or decrease in EKI is accompanied by a significant decrease in triglycerides and cholesterol, zero-, total and NK-Lymphocytes and vagal tone - on the one hand, and increase in sympathetic tone, T-helper and B-lymphocytes level, as well as bactericidity against Staph. aureus - on the other hand. 
In the information space of both discriminant roots, the members of all three clusters are very clearly delineated, as documented by the calculation of Mahalanobis distances (Table 7).

Table 7. Squared Mahalanobis Distances between Clusters of change and F-values $\left(d f=19,5\right.$; for all $\left.p<10^{-6}\right)$

\begin{tabular}{|l|l|l|l|}
\hline $\begin{array}{l}\text { Clusters of } \\
\text { change }\end{array}$ & $\begin{array}{l}\text { EKI } \\
\pm 2,5 \\
\%\end{array}$ & $\begin{array}{l}\text { EKI } \\
>+2,5 \%\end{array}$ & $\begin{array}{l}\text { EKI } \\
<-2,5 \%\end{array}$ \\
\hline EKI $\pm \mathbf{2 , 5}$ & 0 & $\mathbf{2 4}$ & $\mathbf{5 2}$ \\
\hline EKI $>+2,5 \%$ & $\mathbf{1 2 , 9}$ & 0 & $\mathbf{1 3 1}$ \\
\hline EKI $<-2,5 \%$ & $\mathbf{1 0 , 9}$ & $\mathbf{2 3 , 5}$ & 0 \\
\hline
\end{tabular}

Retrospective classification according to the parameters given in table. 8 , infallible.

Table 8. Coefficients and Constants for Classification Functions for Clusters of change in EKI

\begin{tabular}{|c|c|c|c|}
\hline CLUSTERS & $\begin{array}{c}\text { EKI } \\
\pm 2,5 \%(49)\end{array}$ & $\begin{array}{c}\text { EKI } \\
>2,5 \%(19)\end{array}$ & $\begin{array}{c}\text { EKI } \\
<2,5 \%(6)\end{array}$ \\
\hline Variables currently in the model & $\mathrm{p}=, 662$ & $\mathrm{p}=, 257$ & $\mathrm{p}=, 081$ \\
\hline Electrokinetic Index, \% & 0,722 & 6,125 & $-6,745$ \\
\hline Phagocytose Index vs E. coli, \% & $-0,137$ & 0,631 & $-3,391$ \\
\hline Baevsky's Stress Index, units & $-0,001$ & $-0,063$ & 0,099 \\
\hline Leukocytes, $10^{9} / \mathrm{L}$ & $-0,335$ & 0,496 & $-0,081$ \\
\hline ULF HRV PS, $\%$ & $-0,017$ & $-0,073$ & 0,167 \\
\hline 0 Lymphocytes, \% & $-0,037$ & $-0,834$ & 0,508 \\
\hline Immunocytogram Entropy & $-0,0014$ & $-0,1485$ & 0,1086 \\
\hline Mode HRV, msec & $-0,0046$ & $-0,0318$ & 0,0353 \\
\hline AMo/MxDMn Index, units & 0,0002 & 0,0474 & $-0,0642$ \\
\hline$\left(\right.$ VLF+ULF) HRV PS, $\mathrm{msec}^{2}$ & 0,000012 & 0,000094 & $-0,000385$ \\
\hline Bactericidity vs E. coli, $10^{9}$ Bacteria/L & 0,0198 & $-0,0539$ & 0,0936 \\
\hline Polymorphonucleary Neutrophils, \% & 0,050 & 0,955 & $-0,992$ \\
\hline Baevsky's ARS Index, units & $-0,012$ & 0,533 & $-0,930$ \\
\hline Cholesterol standardized, Z & $-0,061$ & 4,270 & $-6,310$ \\
\hline Interleukin-6, ng/L & 0,017 & $-1,838$ & 2,372 \\
\hline Eosinophils, \% & 0,070 & 1,270 & $-1,010$ \\
\hline Popovych's Adaptation Index-2, points & 0,315 & 3,805 & $-3,454$ \\
\hline Panlymphocytes, \% & 0,114 & 0,700 & $-0,793$ \\
\hline Triglycerides standardized, $\mathrm{Z}$ & 0,380 & $-0,848$ & 0,131 \\
\hline Constants & $-0,990$ & $-16,21$ & $-24,53$ \\
\hline
\end{tabular}

\section{CONCLUSION}

Electrokinetic index of buccal epithelium responds to therapeutic factors in different directions, accompanied by characteristic changes in a number of parameters of HRV, immunity and metabolism.

\section{ACKNOWLEDGMENT}

We express sincere gratitude to VY Musiyenko, TA Korolyshyn as well as administration of clinical sanatorium "Moldova" for help in recording tests. 


\section{ACCORDANCE TO ETHICS STANDARDS}

Tests in patients are carried out in accordance with positions of Helsinki Declaration 1975, revised and complemented in 2002, and directive of National Committee on ethics of scientific researches. During realization of tests from all participants the informed consent is got and used all measures for providing of anonymity of participants.

\section{REFERENCES}

1. Babelyuk VYe, Popovych IL, Babelyuk NV, Korolyshyn TA, Dubkova GI, Kovbasnyuk MM, Hubyts'kyi VYo, Kikhtan VV, Musiyenko VYu, Kyrylenko IG, Dobrovolsky YG, Korsuns'kyi IH, Muszkieta R, Zukow W, Gozhenko AI. Perspectives on the use of electrostimulation with the device "VEB" in the management of disorders related to COVID-19. Balneo Research Journal. 2020; 11(3): 328-343.

2. Baevsky RM, Ivanov GG. Heart Rate Variability: theoretical aspects and possibilities of clinical application [in Russian]. Ultrazvukovaya i funktsionalnaya diagnostika. 2001; 3: 106-127.

3. Balanovs'kyi VP, Popovych IL, Karpynets' SV. About ambivalence-equilibratory character of influence of curative water Naftussya on organism of human [in Ukrainian]. Dopovidi ANU. Mat pryr tekhn Nauky. 1993; 3: 154-158.

4. Barylyak LG, Malyuchkova RV, Tolstanov OB, Tymochko OB, Hryvnak RF, Uhryn MR. Comparative estimation of informativeness of leucocytary index of adaptation by Garkavi and by Popovych. Medical Hydrology and Rehabilitation. 2013; 11(1): 5-20.

5. Berntson GG, Bigger JT jr, Eckberg DL, Grossman P, Kaufman PG, Malik M, Nagaraja HN, Porges SW, Saul JP, Stone PH, Van der Molen MW. Heart Rate Variability: Origines, methods, and interpretive caveats. Psychophysiology. 1997; 34: 623-648.

6. Chebanenko OI, Chebanenko LO, Popovych IL. Variety Balneoeffects of Factors Spa Truskavets' and their Forecast [in Ukrainian]. Kyiv. UNESCO-SOCIO; 2012: 496.

7. Douglas SD, Quie PG. Investigation of Phagocytes in Disease. Churchil; 1981: 110.

8. Fajda OI, Hrinchenko BV, Snihur OV, Barylyak LG, Zukow W. What Kerdoe's Vegetative Index really reflects? Journal of Education, Health and Sport. 2015; 5(12): 279-288.

9. Fil' VM, Kyrylenko IG, Popovych IL, Matroshylin AG. Electrokinetic index of buccal epithelium correlated with some functional and metabolic parameters. In: Valeology: the current state, trends and prospects of development. Abstract supplementary XIV International science-practice conf. (Kharkiv-Drogobych, April 14-16, 2016). Kharkiv. VN Karazin NU; 2016: 350-354.

10. Goryachkovskiy AM. Clinical Biochemistry [in Russian]. Odesa. Astroprint; 1998: 608.

11. Gozhenko AI, Korda MM, Popadynets' OO, Popovych IL. Entropy, Harmony, Synchronization, Harmony and Their Neuro-Endocrine-Immune Correlates [in Ukrainian]. Odesa. Feniks; 2021: 232.

12. Heart Rate Variability. Standards of Measurement, Physiological Interpretation, and Clinical Use. Task Force of ESC and NASPE. Circulation. 1996; 93(5): 1043-1065.

13. Honcharenko MS (editor). Valeological toolkit hardware-software diagnostics and monitoring of health [in Ukrainian]. Kharkiv. VN Karazin NU; 2011: 135.

14. Honcharenko MS, Yereshchenko YA. Test system for assessing the physiological state of the body by electrophoretic properties of buccal epithelium cells: Methodical development [in Russian]. Kharkiv; 1992.

15. Kozyavkina NV, Popovych IL, Zukow W. Metabolic accompaniment of thyrotropic effects of bioactive water Naftussya at the women with thyroid hyperplasia. Journal of Health Sciences. 2013; 3(5): 409-424.

16. Kul'chyns'kyi AB, Kovbasnyuk MM, Korolyshyn TA, Kyjenko VM, Zukow W, Popovych IL. Neuro-immune relationships at patients with chronic pyelonephrite and cholecystite. Communication 2. Correlations between parameters EEG, HRV and Phagocytosis. Journal of Education, Health and Sport. 2016; 6(10): 377-401.

17. Kyrylenko IG. Changes in electrokinetic index of buccal epithelium correlated with changes in some parameters of EEG, HRV, hemodynamics and metabolism. Experimental and Clinical 
Physiology and Biochemistry. 2018; 2(82): 5-14.

18. Kyrylenko IG, Fajda OI, Drach OV, Popel SL, Popel RL, Zukow W. Relationships between electrokinetic index of buccal epithelium and some functional and metabolic parameters at men with chronic pyelonephrite. Journal of Education, Health and Sport. 2016; 6(1): 302-314.

19. Kyrylenko IG, Flyunt I-SS, Fil' VM, Zukow W, Popovych IL. Changes in electrokinetic index of buccal epithelium correlated with changes in some parameters of immunity and fecal microbiocenosis. Journal of Education, Health and Sport. 2018; 8(10): 168-170.

20. Kyrylenko IG, Korolyshyn TA, Zukow W, Barylyak LG, Popovych IL. Electrokinetic index of buccal epithelium correlated with some functional and metabolic parameters. In: IX International Symposium "Actual Problems of Biophysical Medicine" (Kyiv, May12-15, 2016). Kyiv. Bohomolets' Institute of Physiology; 2016: 50-51.

21. Lapovets' LYe, Lutsyk BD. Handbook of Laboratory Immunology [in Ukrainian]. L'viv; 2004: $173 \mathrm{p}$.

22. Pat. 2007113, RF. 1994. Device for evaluating the electrokinetic properties of buccal epithelium cells.

23. Pat. 28113, Ukraine, NSI A61V10/00. Method of rapid testing efficiency rehabilitation of health. Shakhbazov VG, Kolupaeva TV, Shuvalov IM et al. 2000; Bul №5.

24. Petsyukh SV, Petsyukh MS, Kovbasnyuk MM, Barylyak LG, Zukow W. Relationships between Popovych's Adaptation Index and parameters of ongoiging HRV and EEG in patients with chronic pyelonephrite and cholecystite in remission. Journal of Education, Health and Sport. 2016; 6(2): 99-110.

25. Popovych IL (editor). General Adaptation Reactions and Body's Resistance in Liquidators of the Chernobyl Accident [in Ukrainian]. Kyiv: Computerpress; 2000: 117 p.

26. Popovych IL, Kul'chyns'kyi AB, Korolyshyn TA, Zukow W. Interrelations between changes in parameters of HRV, EEG and cellular immunity at patients with chronic pyelonephritis and cholecystitis. Journal of Education, Health and Sport. 2017; 7(10): 11-23.

27. Shaffer F, Ginsberg JP. An Overview of Heart Rate Variability Metrics and Norms. Front Public Health. 2017; 5: 258.

28. Shakhbazov VG, Kolupaeva TV, Nabokov AL. New method for determining biological age of man [in Russian]. Laboratornoye delo. 1986; 7: 404-407.

29. Shannon CE. Works on the theory of informatics and cybernetics [transl. from English to Russian]. Moskwa: Inostrannaya literatura; 1963: 329 p.

30. Shkorbatov YG, Kolupaeva TV, Shakhbazov VG, Pustovoyt PA. About relationship electrokinetic properties of nuclei cells of human with physiological parameters [in Russian]. Fiziologiya cheloveka. 1995; 21(2): 25-27.

31. Struk ZD. Multivariable immunotropic effects of bioactive water of Naftussya under drinking monotherapy conditions [in Ukrainian]. Medical Hydrology and Rehabilitation. 2009; 7(2): 9296.

32. Struk ZD. Neuroendocrine and clinical accompaniments of multivariate immunotropic effects of bioactive water Naftussya [in Ukrainian]. Medical Hydrology and Rehabilitation. 2009; 7(4): 5165.

33. Vis'tak HI, Popovych IL. Vehetotropni efekty bioaktyvnoyi vody Naftusya ta yix endokrynnyj i imunnyj suprovody u shhuriv-samok. [Vegetotropic effects of bioactive water Naftussya and their endocrine and immune support in female rats]. Medychna hidrolohiya ta reabilitaciya. [Medical Hydrology and Rehabilitation]. 2011; 9(2): 39-57. [in Ukrainian]. 